\title{
PID Controller Simulator Design for Polynomials Transfer Function
}

\author{
Adri Senen*, Titi Ratnasari, Yoakim Simamora \\ Electrical Engineering Departement, Institute of Technology-PLN, Jakarta, Indonesia
}

\begin{abstract}
PID controller is a controller used to determine precision of a system instrument employing feedback of the system. The component of PID control can be applied simultaneously or singly depends on the response we desired from a plant. However, there is problem in analyzing planned system response; how to get transfer function from a system and analyze it quickly and easily. As of today, control system engineers still encounter difficulty in analyzing system response especially for transfer function using polynomial equations of degree greater than two and employing trial and error method when analyzing response in the simulation. Thus, the writers design a simulator to solve this problem by using Graphical User Interface. The design of the program can be used for polynomial transfer function and installed directly to computer with smaller memory capacity. Hence, it is more user-friendly and not slowing down the operating system of computer. To ensure that the output of simulation correctly generated, we implemented block diagram modeling in simulink. In the future, the result of this research can be implemented as reference to easily understand the characteristics of PID controller especially for polynomials transfer function.
\end{abstract}

Keyword. PID, Controller, simulation, polynomials, response

\section{Introduction}

PID Controller is one of the controllers that widely used in almost $90 \%$ process of industrial system. The system is easily affiliated with other control methods such as Fuzzy and Robust, which makes it better control system. PID Controller consists of the combination of three controllers; P (Proportional) Controller, I (Integral) Controller, and D (Derivative) Controller, and each has its respective tuning parameters to properly operate called constants. Each type has its advantages and disadvantages [3].

It is difficult and boring to view a response of a system with a variety of combination from input signal and control action. The process needs high accuracy and calculation, yet the description of the output oftentimes is inaccurate, especially for polynomial transfer functions [7]. To address this issue, we need simulation software to acquire response from a system with convenience and time efficiency. One of the software that is appropriate for this

*Corresponding author: Ad_Senen@yahoo.com 
simulation is MatLab from Mathworks, Inc. This software is equipped with toolbox to assist the user to do certain calculations. Moreover, a particular toolbox for simulation has been developed called Simulink [9].

Based on the explanation above, the writers design a PID simulator-based graphical user interface which allows displaying a full response of a system with a variety of combination from input signal and control action quickly, easily and accurately. This design can also be used for transfer function in which the degree greater than three. The output of response displayed will be tested and compared with simulation using simulink model and block diagram, as to ensure that the output of the response from the design is generated accordingly.

\section{Designing PID controller}

GUI stands for Graphical User Interface, a display application containing tasks, commands, or components of a program that makes it easy for users to run a program [1]. In this GUI has perfected a Graphical User Interface to make it easier to analyze the PID controller, the view of the GUI can be seen in the image below :

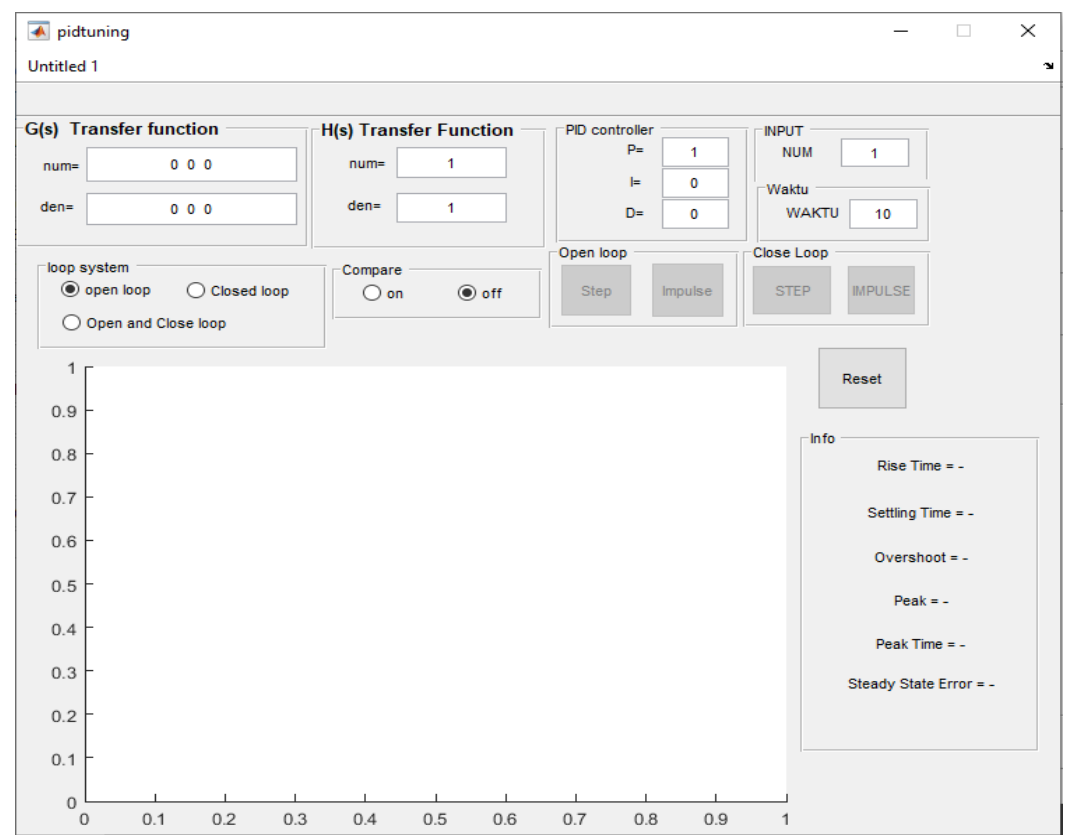

Fig. 1. GUI PID Controller

Before understanding a PID controller, it is necessary to know that the block diagram is used in control. On the GUI PID controller above using block closed loop system diagram

\subsection{Input set point and time}

In the Input Panel and time there are 2 text boxes where the first text box input the desired amount of set point, set point here serves as the size of the controlled value of the desired variable or as input of the control system. In the second edit box is input for the desired time, the time inputs in this condition in seconds, in order to input a variable time is to facilitate the analysis of system response for a certain time. 


\subsection{Loop system}

In a control system, there are two types of control system namely closed loop system and open loop system where in the GUI PID controller that has been created can choose either for closed loop system or open loop system or both.

\subsection{Closed loop system}

The feedback control system is often referred to as a closed-loop control system. Practically speaking, the term feedback control and a closed-loop control can be exchanged for use. In a closed loop control system, the fault signals that work, i.e. the difference between the input signal and the feedback signal, are presented to the controller in such a way as to reduce the error and bring the system output to the desired value. The term closed loop control always means the use of feedback control action to reduce system errors [3].

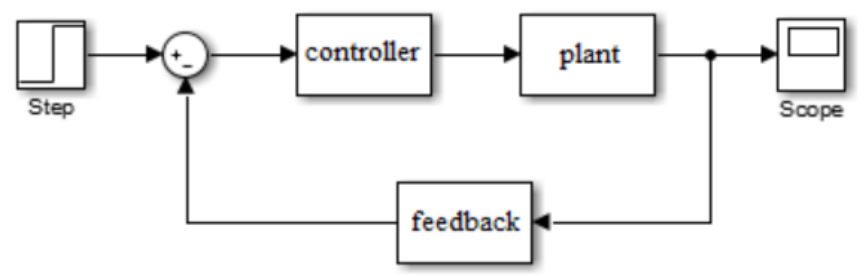

Fig. 2. Closed Loop System Equations

\subsection{Open loop system}

A system whose output has no effect on the control action is called an open-loop control system. In other words, the output open loop control system cannot be used as a feedback comparison with input. In an open loop control system, the output cannot be compared with the reference input. Therefore, for each reference input related to certain operating conditions, as a result, the provision of the system depends on the calibration. In the presence of interference, the open-loop control system cannot perform tasks as expected. An openloop control system can be used only if the connection between the input and output is known and there are no internal or external interference [3]

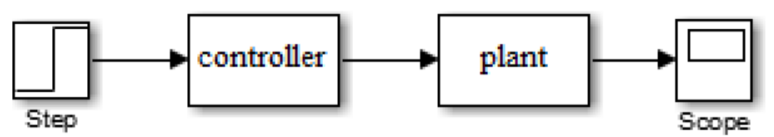

Fig. 3 Open Loop System.

\subsection{Compare}

On the GUI PID of the controller that has been created, there are compare panels or comparators. The Compare Panel serves to compare between graphs so that if you want to line up the graphical response form between the open loop system and the closed loop system can use this panel to see the graphic shape. Not only the open loop system and closed loop system but also can compare between the values of the PID parameter, so it can see the response from each parameter. 


\subsection{Step and impulse input}

There are two types of inputs used in the PID controller GUI, namely the step type input and the impulse type input. Both types of inputs can be used in open loop systems or closed loop systems. Display input in the form of a button as shown below

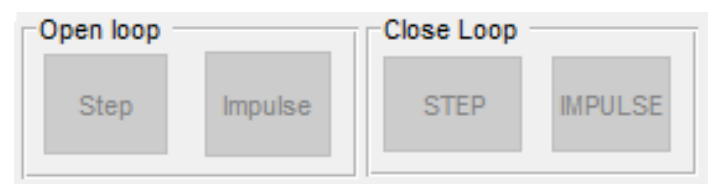

Fig. 4. Open loop system and close loop of panel input system

\subsection{Info}

In this info panel displays the value that need to be analyzed in a control system, the value that need to be analyzed are, Rise time, settling time, Overshoot, Peak, Peak time, Steady State error. This info Panel can be seen in the GUI PID controller as shown below

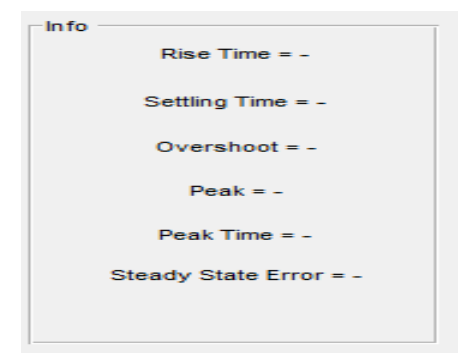

Fig. 5. Bar Info Gui Pid Controller

\section{Simulation and Analysis}

Before designing the PID simulator using a graphic user interface, the first thing to do is to make a block diagram modeling. Modeling is done by taking one of the polynomial equations. The results of this modeling will then be used as a comparison of the design results and test whether the results of the design are correct or not yet

\subsection{Designing PID controller with simulink}

\subsubsection{Proportional - integral controller simulation with simulink}

Polynomial equations for this simulation are

$$
G(s)=\frac{s^{2}+12 . s+15}{s^{3}+3 s^{2}+7 . s+5}
$$


$\mathrm{H}(\mathrm{S})=1 ; \mathrm{Kp}=1 ; \mathrm{Ki}=0.5$; input $=1$; Time $=4$ seconds. The data Input process can be seen in the image:

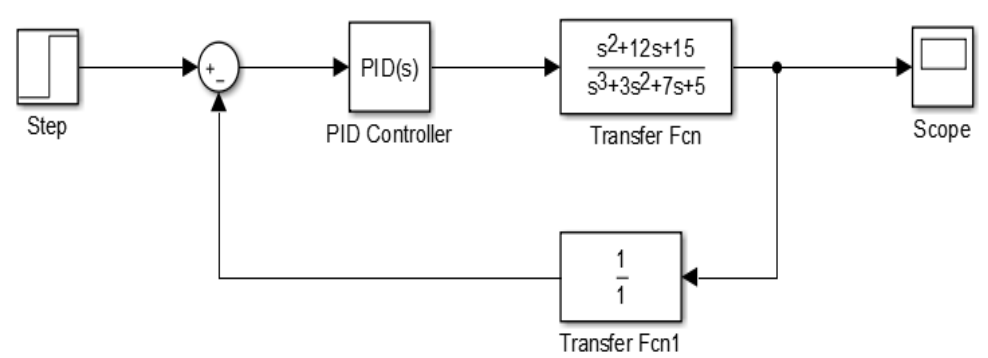

Fig. 6. Block Diagram for proportional integral control

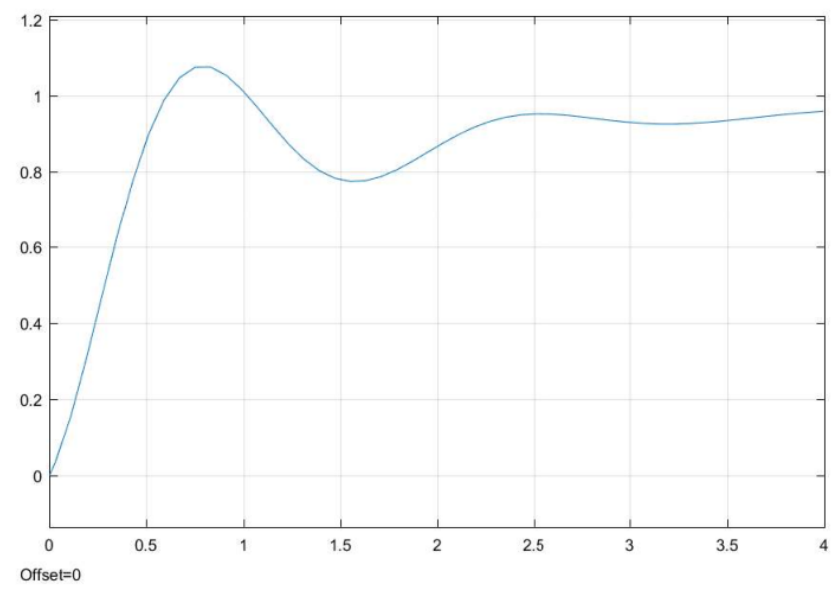

Fig. 7. Output response for $\mathrm{Kp}=1$ and $\mathrm{Ki}=0.5$

System respon from figure 4 shows that proportional integral controls have the characteristic of reducing up time, adding overshoot and down time, as well as eliminating the steady state

\subsubsection{Proportional - integral - derivated controller simulation with simulink}

This simulation uses the following equation:

$$
G(s)=\frac{1}{s^{3}+6 s^{2}+5 s}
$$

$\mathrm{H}(\mathrm{S})=1 ; \mathrm{Kp}=18 ; \mathrm{Ki}=12,811 \mathrm{Kd}=6.3223 ;$ input $=1$. Time $=15$ seconds. The data input process can be seen in the image : 


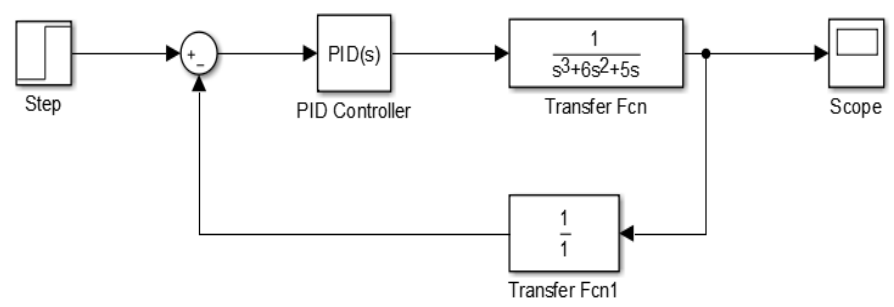

Fig. 8. Block Diagram for Proportional Integral Derivative Control

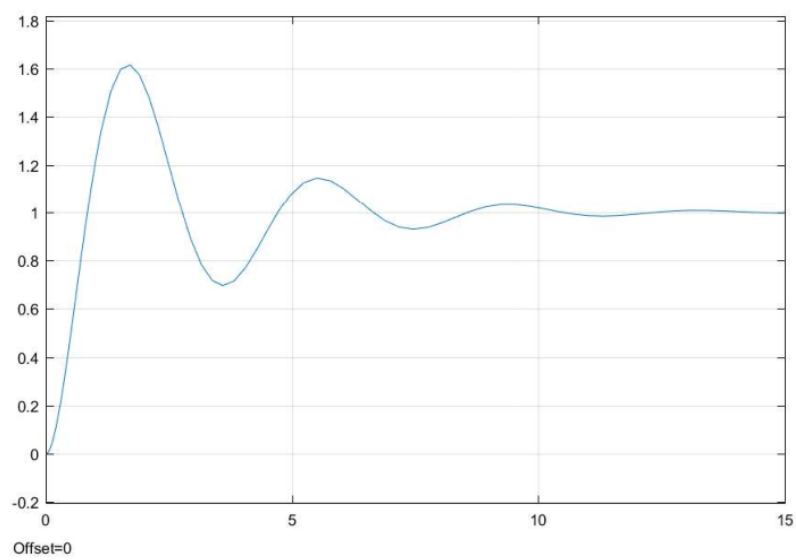

Fig. 9. Process Input data and output response for $\mathrm{Kp}=18, \mathrm{Ki}=12.81138$, and $\mathrm{Kd}=6.3223$

System respon from figure above shows that proportional integral derivative controller have the characteristic of reduce the overshoot, reduce the settling time

\subsection{Designing PID controller with simulink graphic user interface}

\subsubsection{Proportional - integral controller simulation with graphic user interface}

The same simulation is done for proportional and integral controllers by entering the following equation (1). Where $\mathrm{H}(\mathrm{S})=1 ; \mathrm{Kp}=1 ; \mathrm{Ki}=0.5$; input $=1$; Time $=4$ seconds. The data Input process can be seen in the image : 


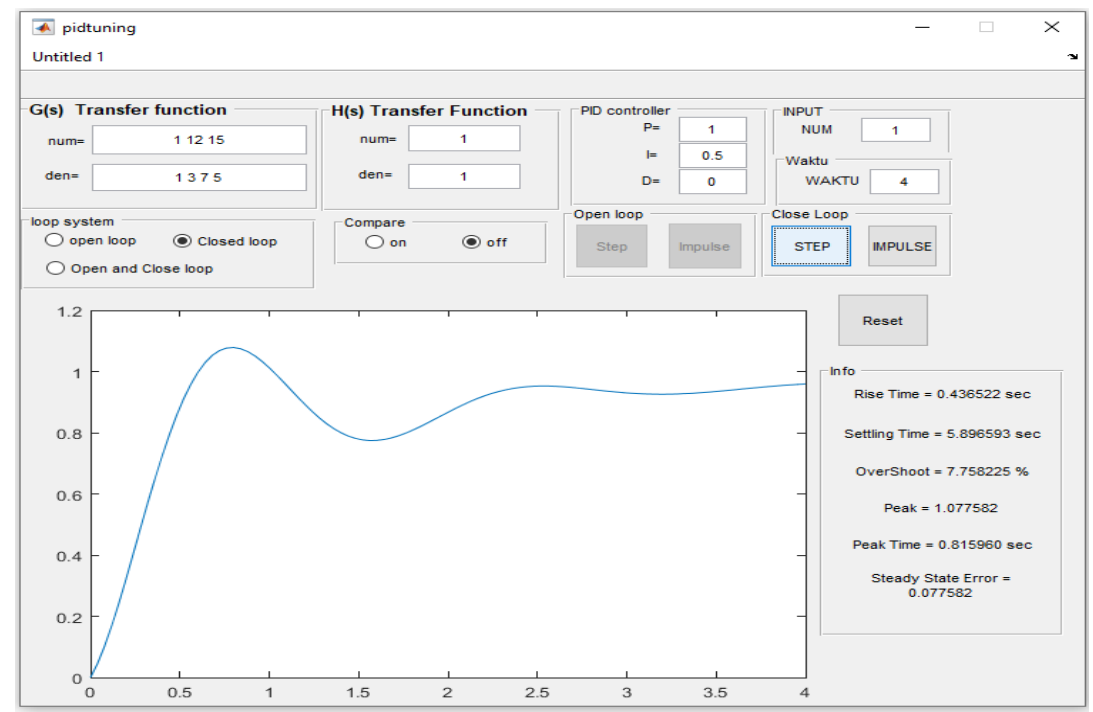

Fig. 10. Process of data input and output response for $\mathrm{Kp}=1$ and $\mathrm{Ki}=0.5$

The results of the simulation respon design for equation (1), show the same output response with the simulation results using simulink based on the block diagram modeling that has been formed before. Integral controls have the characteristic of reducing up time, adding overshoot and down time, as well as eliminating the steady state. Control P and I have the same characteristics in ascending and overshoot time. To be overshoot not excessive the Kp value should be reduced

\subsubsection{Proportional - integral - derivative controller simulation with graphic user interface}

The same thing is done for the PID controller, namely by entering the equation (2). Where $\mathrm{H}$ $(\mathrm{S})=1 ; \mathrm{Kp}=18 ; \mathrm{Ki}=12,811 \mathrm{Kd}=6.3223$; input $=1$; Time $=15$ seconds. The data input process can be seen in the image:

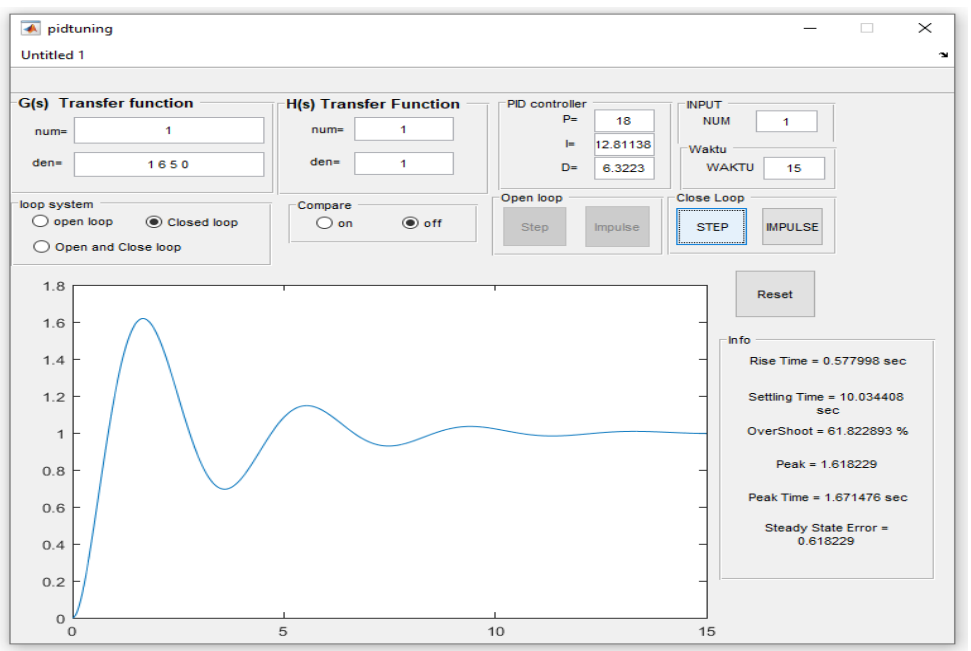

Fig. 11. Process Input data and output response for $\mathrm{Kp}=18, \mathrm{Ki}=12.81138$, and $\mathrm{Kd}=6.3223$ 
The design results show the same response as the response generated by simulink with modeling done with a block diagram by entering the same equation (2). Each of the shortcomings and advantages of each of the P, D controllers and I can cover each other by combining all three in parallel into a proportional controller plus an integral plus differential (PID controller). The elements of the P, I and D controllers overall are aimed at accelerating the reaction of a system, eliminating offsets and generating a large initial change

\section{Conclusion}

The design of PID simulator controller successfully shows that it can be used for polynomial transfer function after experiment on several polynomial equations. Besides, this design is user-friendly and the output can easily enable the user to analyze the response of the system quickly. The accuracy of the simulation is befitting as the output of simulation is similar to the output of tested simulink model (block diagram). This design can be used as software for starter in understanding response of a system in each variance, input, setpoint and each type of controller used. Hence, the information from every function is provided as a whole for user.

The design a PID simulator-based graphical user interface which allows displaying a full response of a system with a variety of combination from input signal and control action quickly, easily and accurately

\section{References}

1. Y. Zhang, Y. Jia, T. Chai, D. Wang, W. Dai and J. Fu, Data-driven PID controller and its application to pulp peutralization process, in IEEE Transactions on Control Systems Technology, vol. 26, no. 3, pp. 828-841, May ( 2018)

2. Ghazanfar Shahgholian, Controller design for three-mass resonant system based on polynomial method, International Journal of Science, Technology and Society. Vol. 5, No. 2, pp. 13-25, (2017)

3. H. J. Live, H. B. Wang, X. M. Zhu, Z. H. Shen and J. Y. Chen, "Simulation Research of Fuzzy Auto-Tuning PID Controller Based on Matlab," IEEE International Conference on Computer Technology, Electronics and Communication (ICCTEC), Dalian, China, , pp. 180-183. (2017)

4. M. A. Siddiqui, S. H. Laskar, M. N. Anwar and M. Naseem, A model-free PI/PID controller based on direct synthesis approach to achieve disturbance rejection, IECON 2019 - 45th Annual Conference of the IEEE Industrial Electronics Society, Lisbon, Portugal, pp. 207-212, (2019)

5. N. A. Patil and G. V. Lakhekar, Design of PID controller for cascade control process using genetic algorithm, International Conference on Intelligent Computing and Control Systems (ICICCS), pp. 1089-1095, Madurai, (2017)

6. Christer Dalen, David Di Ruscio. PD/PID controller tuning based on model approximations: Model reduction of some unstable and higher order nonlinear models. Vol. 38, No. 4, (2017)

7. Hasan, Improvement of systems response of a PID controller in underdamped condition, Systems Response, p. 6, (2015)

8. Hamamci, A New PID tuning method based on transient response control, Balkan Journal of Electrical \& Computer Engineering, Vol.2, No.3, (2014) 
9. L. Ying, The Simulation Design of PID control based on LabVIEW and MATLAB, 2018 International Symposium in Sensing and Instrumentation in IoT Era (ISSI), Shanghai, pp. 1-8, (2018)

10. D William and Dawn Tilbury. Tool Box for MATLAB and Simulink. Addisson Wesley, Inc. (2000)

11. Atherton, D. PI-PD, an Extension of Proportional-Integral-Derivative Control. Measurement and Control, 49(5), 161-165, (2016) 